\title{
Ponctuation et syntaxe en ancien français : Étude d'un corpus de chartes écrites à Liège avant 1292 Méthode de travail
}

\author{
Nicolas Mazziotta \\ Université de Liège \\ Département de Langues et littératures françaises et romanes \\ nicolas.mazziotta@ulg.ac.be
}

\section{Introduction}

Dans cette contribution, nous voudrions présenter la thèse que nous venons de soutenir à l'Université de Liège, intitulée Ponctuation et syntaxe dans la langue française médiévale. Étude d'un corpus de chartes originales écrites à Liège entre 1236 et 1291 (Mazziotta 2007, dir. Marie-Guy Boutier). Ce travail a été mené dans le cadre du projet Khartês, entreprise d'édition et d'étude de chartes originales écrites en français en Wallonie avant 1300 (dir. Marie-Guy Boutier; voir Mazziotta 2004 pour une brève présentation). L'accent sera mis ici sur la méthode que nous avons suivie et la manière dont nous avons mené notre recherche ${ }^{1}$.

La question que nous avons posée d'emblée est la suivante :

Comment, d'après ce qu'on peut observer dans les chartes écrites en français à Liège avant 1292, la ponctuation originale interagit-elle avec la syntaxe dans la langue française médiévale?

Il est inévitable, pour une question de ce type, de partir de faits explicitement attestés dans un ensemble de textes sélectionnés : le corpus. Ce dernier a été déterminé de manière très pratique : nous avons simplement choisis les documents édités dans le cadre du projet Khartês dont la qualité de la transcription diplomatique $^{2}$ était suffisamment fiable pour être exploitée. Nous ne reviendrons pas sur ce choix. En outre, l'emploi d'un corpus - que nous avons voulu électronique - pour traiter un sujet d'ordre linguistique soulève des problèmes relevant de la technique informatique. Nous ne nous y attarderons pas, privilégiant ainsi la dimension proprement scientifique de notre recherche.

Telle qu'elle est formulée, notre question situe d'emblée notre position par rapport à la ponctuation médiévale : nous l'étudions principalement dans ses relations avec la syntaxe et non avec la structure énonciative ou de la sémantique. Cette prise de position sera présentée sous 1. D'autre part, la question que nous avons posée en amène au moins deux autres : Qu'entend-on par ponctuation ? Qu'entend-on par syntaxe? Ces deux questions seront abordées sous 2 . Une fois les notions de base clairement délimitées, nous avons étudié les relations entre la ponctuation et la syntaxe grâce à des outils statistiques. Les étapes et les résultats de cette analyse seront exposés sous 3. 


\section{Position $^{3}$}

Il convient tout d'abord que nous expliquions comment notre question nous mène à prendre position par rapport aux études de nos devanciers. Nous commencerons par mentionner celles de ces dernières qui insistent sur la pluralité des valeurs de la ponctuation (1.1). Nous expliquerons ensuite ce qui a constitué les prémisses de notre travail : le «pari» de la syntaxe (1.2). Dans cette première partie nous utiliserons les termes ponctuation et syntaxe dans leur acception traditionnelle, c'est-à-dire floue. Nous préciserons ces notions infra (2).

\subsection{Pluralité des valeurs de la ponctuation médiévale}

Dès les premières études sur la ponctuation médiévale telle qu'elle est observable dans les textes français, les chercheurs qui se sont penchés sur son fonctionnement ont souligné l'intérêt qu'il y aurait à l'étudier dans ses relations avec, d'une part, la société - habitudes de scribe, suivant les époques, les lieux, etc. (Roques, 1952) - et, d'autre part, les dimensions linguistiques autres que la syntaxe (Marchello-Nizia, 1978). L'idée derrière la recherche des " unités de lecture » (ibid.) est que la ponctuation ne pourrait être décrite de manière satisfaisante que par une approche plurielle. Les études qui ont suivi (en particulier Buridant, 1980 ; Barbance, 1995; Lavrentiev, 2000 ; Baddeley, 2001 ; Mazziotta, 2007b), du moins celles effectuées par des linguistes et des philologues, ont pour la plupart tenté de rendre compte de l'intégration de la ponctuation aux multiples dimensions de la langue, sous ses aspects non seulement morphosyntaxique, mais également énonciatif et sémantique ${ }^{4}$. Schématiquement :

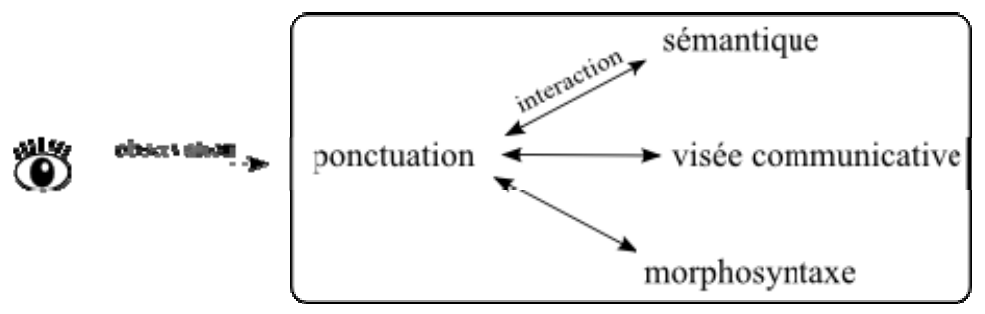

La recherche dans ce sens se poursuit et aboutit au concept d'unité ponctuable, proposé par Alexei Lavrentiev (à paraître), qui suggère une typologie de ces unités et devrait les exploiter pleinement dans la thèse dont il achève actuellement la rédaction.

\subsection{Le « pari » de la syntaxe}

L'approche plurielle n'est toutefois pas la seule qu'il soit possible d'adopter. Pariant sur le fait que la syntaxe permettait tout de même d'expliquer une grande partie de la ponctuation médiévale, Marie-Guy Boutier (2001) a tenté l'expérience d'analyser ainsi l'ensemble des signes d'une charte luxembourgeoise. De cette étude exhaustive, ainsi que de celle que nous avons nous même effectuée ensuite (sous presse) sur un testament de 1272, on peut conclure que le pari est pour l'essentiel gagné pour la grande majorité des signes. Nous avons ensuite comparé la manière dont une construction particulière (protase) était marquée par une ponctuation finale dans un ensemble plus large de documents différents (Mazziotta, 2007c), ce qui a mené à la conclusion que le pari en question continuait à porter ses fruits. Notre thèse se 
situe ainsi dans la continuité de ces études, dont l'objectif est d'épuiser la référence que constitue la syntaxe avant d'exploiter les autres points de vue linguistiques. Schématiquement :

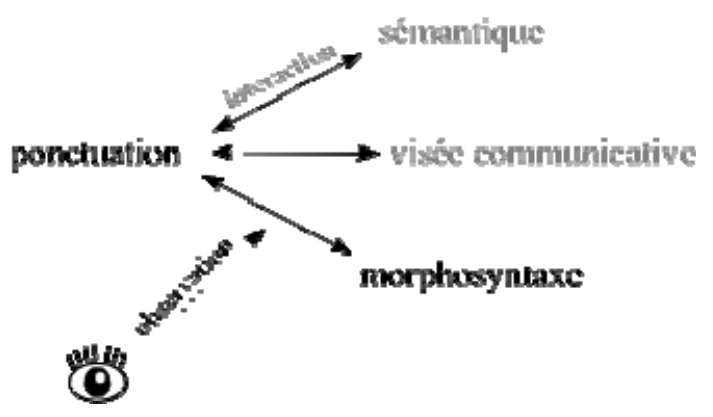

Notre visée n'ignore donc pas que la ponctuation est liée à la dimension énonciative et à la sémantique, mais les relègue temporairement au second plan.

\section{Modèles}

Deux impératifs majeurs s'imposent à l'esprit avec une égale force. Premièrement, si nous voulons évaluer la manière dont ce que nous appelons ponctuation interagit avec ce que nous appelons syntaxe, il est primordial que ces deux concepts soient modélisés de la manière la plus indépendante qui soit. En d'autres termes, nous ne pouvons nous appuyer sur l'un pour définir l'autre et inversement.

D'autre part, notre étude est une recherche effectuée sur un corpus, c'est-à-dire sur des matériaux empiriques. Nous prenons le parti de tirer profit de ces derniers, les modèles construits dont nous rendons compte ci-dessous en sont donc issus et y trouvent leur justification.

Voyons à présent ce qu'est la ponctuation (2.1) et ce qu'est la syntaxe (2.2) pour un certain chercheur qui désire en étudier l'interaction.

\subsection{Qu'est-ce que la ponctuation ? ${ }^{5}$}

Pour définir exactement ce que nous entendons par ponctuation, la première notion à préciser est sans doute celle de langue écrite. Cela sera l'objet de la section 2.2.1. À sa suite, nous montrerons comment le concept de ponctuation peut être progressivement délimité (2.2.2).

\subsubsection{Notion de langue écrite et importance de la spatialité}

Il est fondamental de situer la ponctuation par rapport au système dont elle fait partie et aux autres unités qui composent ce dernier. La question qui a animé les débats depuis que les linguistes se sont à nouveau intéressés à l'écriture est celle de sa relation avec l'oralité. Les deux options suivies, généralement perçues comme contradictoires sont les suivantes : 1/ d'un point de vue phonocentriste (Catach, 2001a et 2001b, p. ex.), la langue écrite est une représentation de la langue orale ; 2/ d'un point de vue autonomiste (Anis, 1983 et 1988, p. ex.), la langue écrite est indépendante de la langue orale. Sans entrer dans le débat, nous considérons que les postulats ne sont pas inconciliables et qu'ils correspondent à des approches 
différentes d'un même objet : les phonocentristes décrivent les relations entre l'écrit et l'oral d'un point de vue linguistique, alors que les autonomistes essayent de produire une description indépendante, ce qui mène à deux descriptions d'égale valeur épistémologique. À nos yeux, c'est la problématique qui permet de déterminer laquelle des deux approches convient. Dans notre cas, l'ancien français n'étant plus parlé par aucun locuteur, il paraît raisonnable d'adopter une attitude autonomiste et de fonder la description sur ce qui fait la spécificité de l'écrit: la spatialité (Harris, 1995, en partic. 45; adopté également par Klinkenberg, 2005).

\subsubsection{Définition progressive des unités de la langue écrite et de la ponctuation}

Le recours à la dimension spatiale de l'écriture, combiné à la dichotomie distinctif/significatif fondatrice de toute sémiotique linguistique (Martinet, 1996, 17 sv.), permet de poser un certain nombre d'oppositions distinguant nettement différents types d'unités.

Tout d'abord, il faut distinguer l'organisation de l'espace des unités organisées, auxquelles nous donnons le nom de grammèmes et que nous notons entre chevrons ${ }^{6}$. Selon le principe susmentionné de la double articulation, ces grammèmes sont soit simplement distinctifs (comme par exemple $<\mathrm{q}>$ ), soit significatifs (comme la chaîne < exemple > ou le kanji $\prec \cdot>$ « eau »). Parmi ces derniers, les linguistes de l'écriture (p. ex. : Anis et coll., 1988) ont pu remarquer que certains d'entre eux ne pouvaient se manifester qu'au travers d'autres unités ; c'est par exemple le cas de la mise en gras ou l'italique. On voit en outre que les unités de deuxième articulation qui servent à graphier les unités signifiantes sont soit organisées séquentiellement (c'est le cas dans les écritures dites "alphabétiques»), soit d'une manière conventionnelle qui dépend de l'unité graphiée; ainsi, l'organisation des traits dans l'abréviation médiévale $\langle\square\rangle$ pour < et > est propre à ce grammème. Enfin, il faut distinguer les unités de ce dernier type qui peuvent être paraphrasées par une autre unité significative, comme c'est le cas pour l'abréviation que nous venons mentionner, de celles qui ne le peuvent pas, que nous nommons ponctogrammes.

Une fois toutes ces unités définies, nous pouvons enfin délimiter exactement, le moins intuitivement possible, notre propre acception du mot ponctuation : «ensemble des ponctogrammes d'une langue écrite spécifique ». Dans cette définition, le terme ponctogramme désigne une unité minimale de la langue écrite n'organisant pas l'espace (grammème), exprimant un contenu, ne dépendant pas matériellement d'une autre unité, construite à l'aide de traits qui ne se combinent pas obligatoirement sur un même axe et non paraphrasable par d'autres unités significatives. Schématiquement, la progression de la délimitation du concept est la suivante (de gauche à droite) :

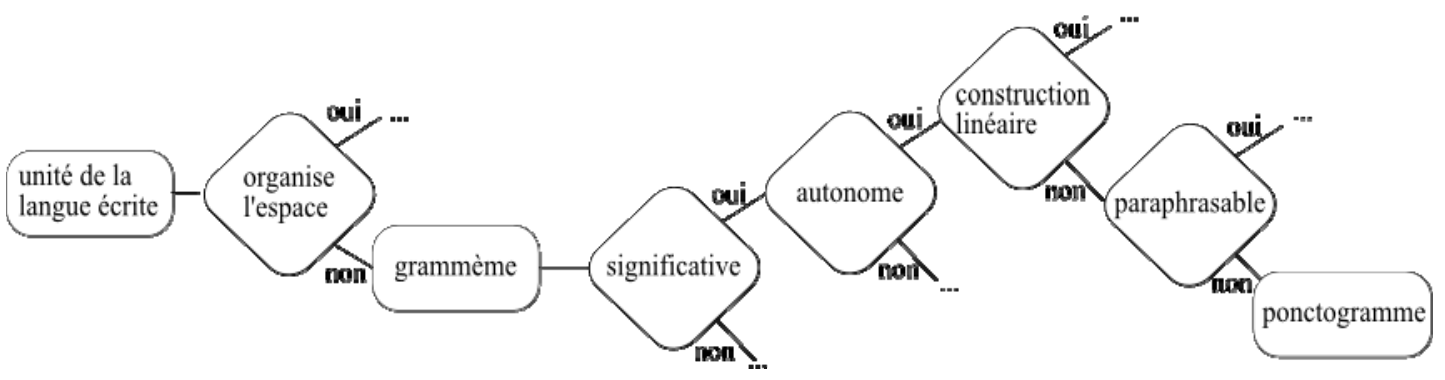


Nous avons donc à notre disposition un arbre décisionnel qui nous permet, pour chaque grammème rencontré, de déterminer s'il s'agit ou non d'un ponctogramme. Ainsi, dans ${ }^{7}$,

et le doit sires giles et sa mere loer en toz líus la uliglise vorrat ; et por ce [12] ke ce soit ferme et|stable auonos a ces letres pendut notre saeal (Mai 1236, Archives de l'État à Liège, Cathédrale Saint-Lambert à Liège, ligne 11)

le grammème souligné a été reconnu comme un ponctogramme, en vertu du fait qu'il n'organise pas l'espace, est plus que distinctif (il comporte un surcroît de sens), est autonome (par rapporta à la substance des autres grammèmes), n'est pas construit sur le même axe que celui sur lequel il se combine avec les autres grammèmes (le point et la virgule sont superposés et non enchaînés horizontalement) et il n'est pas possible de le paraphraser par des mots. On pourrait faire le même exercice avec chacune des unités graphiques du corpus et la ranger ainsi dans la classe des ponctogrammes ou non. Cette démarche déductive part d'une définition en intension de la ponctuation. Employer le terme ponctuation ne peut se faire qu'à la fin d'un exposé passant en revue tous les oppositions impliquées. Il faut par ailleurs remarquer qu'ainsi défini, le concept de ponctuation ne recouvre pas les «majuscules» et les phénomènes d'agglutination de mots.

Enfin, on constate que l'objectif fixé au début de la section 2 est bien rempli : nous ne nous sommes pas servi de la syntaxe pour définir la ponctuation. Plus généralement, la définition obtenue ne postule aucune fonction particulière attribuée aux ponctogrammes.

\subsection{Qu'est-ce que la syntaxe ?}

Définir le contenu du terme syntaxe est une entreprise extrêmement délicate, du fait de la multiplicité des approches déjà existantes et de l'importance de la tradition dans ce domaine. Il est cependant nécessaire de faire un effort de systématisation, car les grammaires de l'ancienne langue (Moignet, 1988 ; Ménard, 1994 pour la syntaxe; Buridant 2000), aussi excellentes qu'elles soient, sont soit tributaires de la grammaire traditionnelle, soit hétérogènes. Elles ne permettent pas d'étudier systématiquement un texte ou un corpus de manière exhaustive. Il nous faudra en premier lieu distinguer nettement la syntaxe de la morphologie (2.2.1). Ensuite, nous ferons une brève présentation des principaux constituants que nous avons définis (2.2.2).

\subsubsection{Opposition morphologie vs syntaxe et niveaux de l'analyse syntaxique}

La première difficulté consiste à défaire le nœud constitué par le mot morphosyntaxe. Distinguer la morphologie de la syntaxe n'est pas chose aisée et nécessite une prise de position. Nous limitons l'acception de syntaxe au sens particulier «ensemble des règles de dépendance et de coocurrence des constituants ", alors que la morphologie est reléguée à un système de marques, relevant exclusivement du plan de l'expression. Nous verrons ci-dessous un exemple concret qui permet de comprendre cette conception.

En outre, la syntaxe peut jouer à différents niveaux (Lazard, 1984). On peut ainsi distinguer une syntaxe dite de mot, une syntaxe immédiate et une syntaxe argumentale. La première concerne les relations entre les éléments de formation des mots, la deuxième celles entre les constituants immédiats du syntagme, la troisième les relations entre les constituants immédiats des propositions. 
Cette tripartition posée, nous pouvons montrer comment, au niveau de la syntaxe de mot, on distingue la syntaxe de la morphologie. Ainsi, la relation qui unirait $i l$ à dit dans $i l$ dit est une relation syntaxique entre le «sujet» et le "verbe", alors que les marques segmentales de cette relation (accord et forme du pronom) relèvent de la morphologie.

\subsubsection{Types de constituants}

La définition du concept passe idéalement par celle de toutes les structures et de toutes les relations syntaxiques possibles. Notre question s'appliquant à un corpus, cet effort a dû être fourni pour que l'annotation exhaustive du corpus puisse être envisageable. Dans cette optique, nous avons suivi la théorie de la «relation minimale » d'Alain Lemaréchal (1997), qui s'est révélée particulièrement efficace. Ce n'est pas le lieu d'un exposé détaillé à ce propos; retenons simplement, pour la bonne compréhension de l'exposé, que nous avons été amené à (re)définir un certain nombre d'unités comme suit.

La proposition est définie comme une structure syntaxique organisée autour d'un verbe qui a la fonction de prédicat et qui porte des marques de temps, de mode et d'aspect (catégories verbales) ${ }^{9}$. Les constituants qui gravitent autour du prédicat sont ses arguments, ce qui inclut les actants (dont la forme est déterminée par le prédicat) et les circonstants $^{10}$. La phrase est définie de manière empirique ${ }^{11}$ comme une proposition (dont le verbe est conjugué à un mode personnel) qui joue le rôle d'énoncé, c'est-à-dire qui exprime un message avec une certaine visée communicative ${ }^{12}$.

Les relateurs sont des constituants dont le sens modifie une relation qu'ils ne contractent pas eux-mêmes, mais qui lie le constituant dans lequel ils sont intégrés à un autre constituant de même niveau - on parle, selon la terminologie d'Alain Lemaréchal (1997), de spécification. Ainsi dans la phrase

La afaita [«transférer (un droit réel immobilier) à (un bénéficiaire) devant la juridiction compétente et selon les formes prescrites »] ilh Werris devant diz a sangor Arnult devant nomet ii verges [7] et demie d'aluez (29 octobre 1267, Archives de l'État à Liège, Abbaye du Val-Benoît à Liège, ligne 6)

le relateur $a$ fait partie de l'actant a sangor Arnult et précise que ce dernier joue le rôle sémantique de bénéficiaire de l'action exprimée par le verbe afaita, qui a la fonction de prédicat. C'est donc la relation de l'ensemble de l'actant au prédicat qui est spécifiée sémantiquement et non une relation qui n'impliquerait que le relateur. Lorsqu'un relateur lie deux constituants de même fonction, nous l'appelons coordonnant.

\section{Résumé des analyses et résultats ${ }^{13}$}

Les modèles présentés sous 2 sont exploitables pour annoter le corpus en vue d'une extraction automatisée des structures et constituants syntaxiques ainsi que des ponctogrammes. Après avoir extrait les données de notre corpus, il est nécessaire de les traiter. Pour ce faire, nous avons choisi de faire usage des statistiques et d'appliquer une démarche progressive (3.1 à 3.3). Nous ne reporterons pas ici de résultats chiffrés, car leur intérêt serait très faible s'ils étaient présentés indépendamment de l'ensemble des tests effectués.

\subsection{Première analyse : réduction des données, présence des ponctogrammes ${ }^{14}$}

Tout d'abord, pour rentabiliser l'outil choisi, il est nécessaire de réduire les données à un ensemble restreint de variables : chaque constituant se voit défini par six caractéristiques morphosyntaxiques et 
positionnelles que nous avons jugées fondamentales pour les décrire. Ces variables répondent à six questions : 1/ du point de vue de l'ordre linéaire des mots, le constituant est-il le premier de la structure qu'il sert à construire (abrégé $L I D$ dans le tableau ci-dessous) ? 2/ le constituant est-il le dernier de la structure qu'il sert à construire $(L I F)$ ? 3/ quelle est la nature et le niveau d'intégration $(M I)$ syntaxique de la structure qui le contient? 4/ quelle est la fonction du constituant $(M F)$ ? 5/ est-il de nature propositionnelle, de mode personnel ou non $(M N)$ ? 6/ est-il relaté $(M R)$ ? Par exemple, en ce qui concerne constituants immédiats de la phrase suivante,

- en tiesmong de ceste [8] chose $\cdot$ aj io fait ces letres saieler de mon|saiel $\cdot$ lan del jncarnation jhesu crist $\cdot \mathrm{mil} \cdot \mathrm{et} \cdot \mathrm{cc} \cdot \mathrm{et} \cdot \mathrm{xxx} \cdot \mathrm{vj} \cdot[9]$ el mois $\cdot$ de jvle (Mai 1236, Archives de l'État à Liège, Cathédrale Saint-Lambert à Liège, ligne 7)

les informations accessibles peuvent être résumées au tableau :

\begin{tabular}{lllll} 
Texte & MI & MF & MN & MR LID LIF \\
\hline en tiesmong de ceste chose & phrase & circonstant & non prop. & oui oui non \\
aj & phrase & prédicat & non prop. & non non non \\
fait & phrase & prédicat & non prop. & non non non \\
io & phrase & sujet & non prop. & non non non \\
ces letres saieler de mon $\mid$ saiel & phrase & régime & prop. non pers. & non non non \\
lan $[\ldots] \cdot$ mil $\cdot$ et $\cdot$ cc $\cdot$ et $\cdot$ xxx $\cdot v j$ & phrase & circonstant & non prop. & non non non \\
el mois $\cdot$ de jvle & phrase & circonstant & non prop. & oui non oui
\end{tabular}

Une fois ces informations recueillies, il est possible de mettre en relation les réponses à ces questions et la simple présence de ponctuation de part et d'autre des constituants, sans tenir compte, dans un premier temps, de la forme des ponctogrammes. Pour ce faire, les techniques statistiques les plus classiques en sciences humaines (analyse des tableaux de contingence à l'aide du test du chi-deux ${ }^{15}$ ) permettent d'entamer la recherche. L'objectif de l'opération est de sélectionner, au départ d'une très grande quantité de données, les associations (attractions ou répulsions) statistiquement significatives entre une des modalités de ces variables et la présence d'un ponctogramme.

Néanmoins, la méthode (non les tests choisis) se révèle inefficace. Ainsi, il est vrai que nous pouvons, par exemple, constater que les constituants non relatés attirent plus la ponctuation à l'initiale que les constituants relatés, alors que les circonstants attirent le marquage de part et d'autre. Mais que dire d'un circonstant relaté par rapport à un autre qui ne le serait pas? L'atomisation en six variables des individus soumis à l'analyse rend ce genre de question complètement insoluble. Au terme de cette première étape, nous obtenons un ensemble inanalysé de données... 


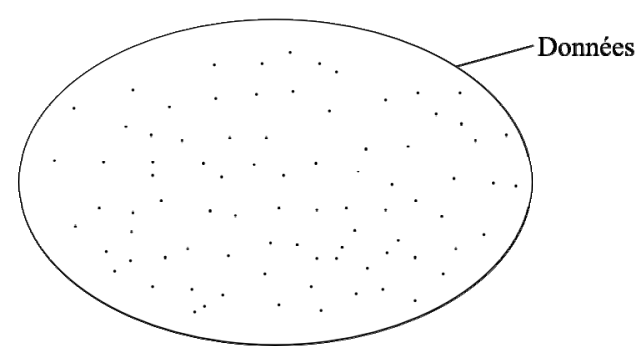

Il faut donc s'employer à trouver le moyen d'envisager simultanément toutes les variables morphosyntaxiques et positionnelles, ce qui est relativement aisé si l'on regroupe toutes les variables citées ci-dessus en une seule « super variable ». La quantité de modalités de cette dernière est très élevée et le discernement des attractions/répulsions significativess entre structures et ponctogrammes doit être évaluée par une série de chi-deux successifs ${ }^{16}$. Ces nouveaux dépouillements montrent, au milieu de la masse de constituants inégalement marqués par la présence d'un ponctogramme, ceux dont le marquage ou le rejet du marquage a la plus faible probabilité d'être dû au hasard. Ce qui ressort de cette première étape, où les données sont réduites à une représentation très abstraite, c'est une liste de points forts concernant : 1 / la différence de fréquence entre le marquage de la phrase et celui des autres propositions ; 2/ la spécificité du marquage d'un certain nombre de types d'arguments; 3/ le rejet manifeste du marquage du prédicat ; 4/ la faible fréquence de marquage à la suite des relateurs ; $5 /$ la forte présence de marquage devant les coordonnants. Schématiquement :

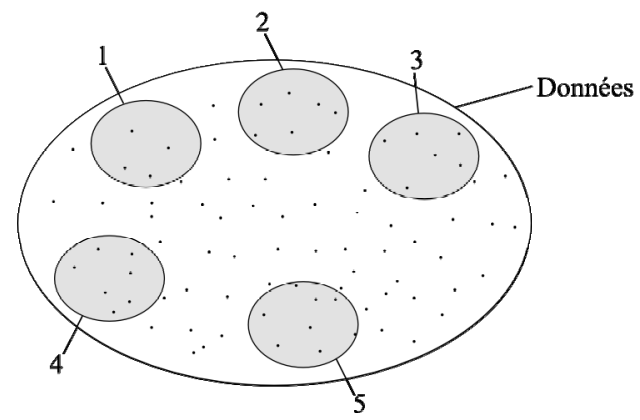

Comme nous l'avons représenté sur ce schéma, la sélection d'un ensemble de points forts se fait en laissant de côté les individus pour lesquels aucune tendance forte ne semble se détacher. Cette réduction supplémentaire de l'information est nécessaire si nous voulons nous assurer que les faits sur lesquels nous travaillons ont peu de chance d'être dus à des causes aléatoires.

\subsection{Focalisation sur les points forts ${ }^{17}$}

Le débroussaillage effectué, ces grandes lignes sont inspectées de manière plus concrète : pour chaque tendance qui le justifie, nous calculons la probabilité que l'attraction ou la répulsion observée soit généralisée. Il en ressort que la plupart des tendances observées étaient générales - ou trop faiblement illustrées pour être évaluées de ce point de vue. Par ailleurs, en mettant en relation la ponctuation avec le contexte immédiat, on observe que beaucoup de constituants sont davantage, voire exclusivement marqués au contact d'autres constituants attirant également le marquage ou dans un contexte de coordination. Ainsi, la ponctuation n'est pas obligatoire, mais sa présence est certainement liée à un 
contexte syntaxique spécifique. Cet examen détaillé des tendances mises en évidence permet en fin de compte de faire le tri parmi les tendances et de repérer celles qui sont manifestement dues à l'entourage du constituant ou au document dans lequel il est attesté.

\subsection{La question de la forme d'expression ${ }^{18}$}

De cette étude de la fréquence du marquage ressort un ensemble d'environnements propices à la présence de ponctuation, mais nous ne savons rien sur l'association éventuelle entre la forme des ponctogrammes et les structures syntaxiques. À ce moment, il est possible de réintroduire les considérations portant sur la forme des ponctogrammes et d'employer l'Analyse Factorielle des Correspondances (AFC) pour décrire les données. En nous focalisant uniquement sur les attractions repérées à l'aide des méthodes présentées ci-dessus (3.1), nous partons donc d'un tri croisé pour mesurer les associations entre la forme des ponctogrammes et la tendance au marquage spécifique à la position où se trouve ce ponctogramme (ce qui inclut l'absence d'environnement attirant le marquage). Après une analyse exploratoire, il est utile de compléter l'étude par une série de tests évaluant la probabilité que les regroupements entre la forme des ponctogrammes et l'environnement dans lequel on les rencontre soit due au hasard. Dans la majorité des cas observés, les contrastes mis en évidence par l'AFC correspondent à des oppositions significatives. L'étude détaillée de la forme mène à la conclusion suivante : les ponctogrammes autres que $\langle\cdot\rangle$ sont plus rares (essentiellement sous les formes $\langle/\rangle,\langle\lrcorner\rangle,\langle\zeta\rangle,\langle\boldsymbol{\Phi}\rangle,\langle\cdot \cdot\rangle,\langle/ \mid\rangle,\langle:\rangle$ et $\langle;\rangle)$, et leur emploi paraît plus spécifique à un environnement donné. En d'autres termes : non seulement les scribes ne ponctuaient pas n'importe où, mais, en plus, ils n'employaient pas indifféremment les signes.

Les méthodes ne permettant pas de traiter de manière efficace les ponctogrammes peu attestés, il est utile de compléter l'analyse outillée par un commentaire, laissant de côté les statistiques pour une étude plus philologique. Ces observations mènent, d'une part, à la critique de la validité de la transcription : 1/ certaines distinctions entre les formes sont peut-être superflues; 2/ certaines unités peuvent être confondues avec d'autres. D'autre part, la forme des ponctogrammes pose la question de la relation entre les ponctogrammes et le reste du système graphique.

\section{Porte ouverte}

Nous avons ainsi montré comment notre travail s'est développé, partant d'une question précise (0) dans une problématique définie (1), construisant les outils conceptuels pour y répondre (2) et exploitant finalement les matériaux de manière systématique et exhaustive (3).

En somme, la méthode a porté ses fruits : il est clair que la ponctuation peut être décrite en grande partie par la syntaxe. Toutefois, en observant plus intuitivement les attestations, nous repérons également, comme nous nous y attendions, un certain nombre de tendances liées à des facteurs étrangers à la morphosyntaxe : la ponctuation de formules spécifiques au type discursif, celle des chiffres ou encore la présence d'un ponctogramme devant les noms de personnes. L'aspect morphosyntaxique épuisé, il est à présent possible de nous tourner vers les aspects énonciatifs et sémantiques. Néanmoins, nous ne voudrons pas les exploiter comme palliatifs aux endroits où rien d'autre ne fonctionne pour expliquer la ponctuation, mais plutôt les observer en système, dans leurs interactions avec le point de vue morphosyntaxique. 


\section{Références}

Abdi, H. (2007). Bonferroni and Šidák corrections for multiple comparisons. Salkind, 2007.

Anis, J. (1983). Pour une graphématique autonome. Langue française, 59, 31-44.

Anis, J. (1988). Une graphématique autonome ? dans Catach, 1988, 213-223.

Anis, J. et coll. (1988). L'écriture. Théories et descriptions. Bruxelles : De Boeck .

Baddeley, S. (2001). La ponctuation de manuscrits français du IX ${ }^{\mathrm{e}}$ au XII ${ }^{\mathrm{e}}$ siècle. Biedermann-Pasques et coll., 2001, 139-149.

Barbance, C. (1995). La ponctuation médiévale : quelques remarques sur cinq manuscrits du début du XV $\mathrm{XV}^{\mathrm{e}}$ siècle. Romania, 113, 505-527.

Biedermann-Pasques, L. et coll. (2001). Usages graphiques, normes et ponctuation. Paris : CNRS.

Boutier, M.-G. (2001). Études sur des chartes luxembourgeoises. Gärtner et coll., 2001, 419-447.

Buridant, Cl. (1980). Le strument et et ses rapports avec la ponctuation dans quelques textes médiévaux. DessauxBerthonneau, 1980, 13-53.

Catach, N. (1988). Pour une théorie de la langue écrite. Actes de la Table Ronde internationale C.N.R.S.-H.E.S.O. Paris, 23-24 octobre 1986. Paris : CNRS.

Catach, N. (2001a). Graphétique. Holtus et coll., 2001, 725-735.

Catach, N. (2001b). Graphémique. Holtus et coll. 2001, 736-747.

Dessaux-Berthonneau, A.-M. (1980). Théories linguistiques et traditions grammaticales. Lille : Presses universitaires de Lille.

Gärtner, K. et coll. (2001). Skripta, Schreiblandschaften und Standardisierungstendenzen. Trier : Kliomedia.

Hagège, Cl. (1986). L'homme de paroles. Contribution linguistique aux sciences humaines. Paris : Fayard.

Harris, R. (1995). Signs of writing. London/New-York : Routledge.

Holtus, G. et coll. (2001). Lexicon der romanistischen Linguistik 1(1). Tübingen : Niemeyer.

Howell, D. C. (1998). Méthodes statistiques en sciences humaines. Paris/Bruxelles : De Boeck et Larcier.

Klinkenberg, J.-M. (2005). Vers une typologie des fonctions de l'écriture. De la linéarité à la spatialité. Bulletin de la Classe des Lettres, 6(16), 157-196.

Lavrentiev, A. (2000). À propos de la ponctuation dans l'Image du monde. La licorne, 52, 23-35.

Lavrentiev, A. (2007). Actes de la journée d'étude sur les systèmes graphiques de manuscrits médiévaux et incunables français : ponctuation,segmentation, graphies. Lyon, ENS-LSH, 6 juin 2005. Chambéry : Éditions de l'université de Savoie.

Lavrentiev, A. (à paraître). Pour une méthodologie d'étude de la ponctuation médiévale basée sur une approche typologique. Verbum.

Lazard, G. (1984). La distinction entre nom et verbe en syntaxe et en morphologie. Modèles linguistiques, 6(1), 2939.

Lazard, G. (1994). L'actance. Paris : Presses Universitaires de France.

Lemaréchal, A. (1997). Zéro(s). Paris : Presses Universitaires de France. 
Marchello-Nizia, Chr. (1978). Ponctuation et « unités de lecture » dans les manuscrits médiévaux ou : je ponctue, tu lis, il théorise. Langue française, $40,32-44$.

Martinet, A. (1996). Éléments de linguistique générale. Paris : Armand Colin.

Mazziotta, N. (20007a). Ponctuation et syntaxe dans la langue française médiévale. Étude d'un corpus de chartes originales écrites à Liège entre 1236 et 1291. Liège : Université de Liège (thèse de doctorat préparée sous la direction de Marie-Guy Boutier).

Mazziotta, N. (2007b). Inconstance ou consensus ? Marquage de la protase en tête de phrase dans les chartes liégeoises du $13^{\mathrm{e}}$ siècle. Trotter, 2007, t. 2, 225-238.

Mazziotta, N. (2007c). Ponctuation et parties du discours diplomatique dans les actes émanés de la cour allodiale de Liège entre 1260 et 1290. Lavrentiev, 2007, 67-84.

Mazziotta, N. (sous presse). Étude linguistique de la ponctuation d'une charte originale en langue française (Liège 1272. Orbis.

Ménard, Ph. (1994). Syntaxe de l'ancien français. Bordeaux : Bière.

Moignet, G. (1988). Grammaire de l'ancien français. Morphologie - Syntaxe. Paris : Klincksieck.

Pellat, J.-Chr. (1998). Indépendance ou interaction de l'écrit par rapport à l'oral ? Recensement critique des définitions du graphème. Catach, 1988, 133-146.

Roques, M. (1952). Le manuscrit fr. 794 de la Bibliothèque Nationale et le scribe Guiot. Romania ,73, 177-199.

Salkind, N. J. (2007). Encyclopedia of Measurement and Statistics. Thousand Oaks : Sage.

Trotter, D. (2007). Actes du XXIV Congrès International de Linguistique et de Philologie Romane, Aberystwyth, 1-6 août 2004. Tübingen : Niemeyer.

1 Un résumé de la thèse (développé dans la présente contribution) ainsi qu'une table des matières sont accessibles en ligne : http ://bictel.ulg.ac.be.

2 Soit une édition qui se veut la moins interprétative possible, préservant les tracés originaux de certains caractères, la ponctuation originale, etc.

3 Cf. Mazziotta, 2007, § 0.1.1.

4 La tripartition, adoptée également par Ludmilla Védénina pour l'analyse de la ponctuation moderne (1989), est héritée du fonctionnalisme praguois et se retrouve actualisée dans la théorie des trois points de vue, développée en France par Gilbert Lazard et Claude Hagège (voir notamment Hagège 1985).

5 Cf. Mazziotta, 2007, ch. 2.

6 Le terme graphème étant particulièrement polysémique (Pellat 1988), nous avons préféré nous en débarrasser complètement. L'élaboration d'une terminologie complète de toutes les unités de la langue écrite a été nécessaire (voir Mazziotta, 2007, ch. 2). Sa présentation alourdirait considérablement l'exposé ; nous nous en passerons donc. 
7 Cette transcription «diplomatique » préserve la ponctuation médiévale et les oppositions originales entre $\langle i\rangle$ et $\langle j\rangle$ et $\langle u\rangle$ et $\langle v\rangle$. Les espaces que nous avons jugées plus brèves qu'une espace normale sont indiquées par \langle\rangle . Aucune majuscule ou ponctuation moderne n'a été introduite.

Cf. Mazziotta, 2007, ch. 3.

$9 \quad$ Cette définition inclut les structures organisées autour d'un verbe conjugué à un mode non personnel (infinitif ou participe). Nous parlons ainsi de propositions participiales ou infinitivales.

10 Ce classement des arguments est proche de celui qu'a proposé Gilbert Lazard $(1994,81)$.

11 Le travail de segmentation du texte en phrases est facilité par le fait que l'ancien français est une langue où la «phrase non verbale » est rare et son emploi limité à certains contextes d'énonciation spécifiques (Ménard, 1994, §203; Moignet, 1988, 97).

12 Cette définition est en partie fondée sur la théorie des trois points de vue (voir note 4).

13 Cf. Mazziotta, 2007, ch. 4 à 7.

14 Cf. Mazziotta, 2007, ch. 5.

15 Ce test (voir Howell, 1998 pour une présentation simple) doit être complété par des ajustements (voir note 16) ou suppléé par un test exact de Fisher en fonction des données qui lui sont soumises.

16 En l'occurrence, l'ajustement de Šidák (voir Abdi, 2007).

17 Cf. Mazziotta, 2007, ch. 6.

18 Cf. Mazziotta, 2007, ch. 7. 